\title{
Approximate Analytical Solutions of the Fractional-Order Brusselator System Using the Polynomial Least Squares Method
}

\author{
Constantin Bota and Bogdan Căruntu \\ Department of Mathematics, "Politehnica" University of Timişoara, Piaţa Victoriei 2, 300006 Timişoara, Romania \\ Correspondence should be addressed to Bogdan Căruntu; bogdan.caruntu@upt.ro
}

Received 31 July 2014; Revised 11 November 2014; Accepted 11 November 2014

Academic Editor: Dumitru Baleanu

Copyright (C) 2015 C. Bota and B. Căruntu. This is an open access article distributed under the Creative Commons Attribution License, which permits unrestricted use, distribution, and reproduction in any medium, provided the original work is properly cited.

The paper presents a new method, called the Polynomial Least Squares Method (PLSM). PLSM allows us to compute approximate analytical solutions for the Brusselator system, which is a fractional-order system of nonlinear differential equations.

\section{Introduction}

In recent years, in many practical applications in various fields such as physics, mechanics, chemistry, and biology (see, e.g., [1-6]), the problems being studied are modeled using fractional nonlinear equations.

For most of such fractional nonlinear equations, the exact solutions cannot be found and, as a consequence, a numerical solution or, if possible, an analytical approximate solution of these equations is sought.

Due to the complexity of this type of problems, a general approximation algorithm does not exist and, thus, various approximation methods, each with its strong and weak points, were proposed, including, among others:

(i) the Adomian Decomposition Method [7-9],

(ii) the Homotopy Analysis Method [10-12],

(iii) the Homotopy Perturbation Method [13, 14],

(iv) the Laplace Transform Method [15, 16],

(v) the Fourier Transform Method [17],

(vi) the Variational Iteration Method [18-20].

The objective of our paper is to present the Polynomial Least Squares Method (PLSM), which allows us to compute approximate analytical solutions for the Brusselator system.
The fractional order Brusselator system was recently studied by several authors [21-23] and can be expressed as follows.

We consider the following Brusselator system:

$$
\begin{gathered}
D_{t}^{\alpha_{1}} x(t)=a-(\mu+1) \cdot x(t)+x(t)^{2} \cdot y(t), \\
D_{t}^{\alpha_{2}} y(t)=\mu \cdot x(t)-x(t)^{2} \cdot y(t),
\end{gathered}
$$

together with the initial conditions:

$$
x(0)=c_{1}, \quad y(0)=c_{2},
$$

where $a>0, \mu>0,0<\alpha_{1} \leq 1,0<\alpha_{2} \leq 1, c_{1}, c_{2}$ are real constants, and $D_{t}^{\alpha}$ denotes Caputo's fractional derivative [15]:

$$
D_{t}^{\alpha}=\frac{1}{\Gamma(1-\alpha)} \cdot \int_{0}^{t}(t-\zeta)^{-\alpha} \cdot x^{\prime}(\zeta) d \zeta, \quad 0<\alpha \leq 1
$$

In the next section we will introduce PLSM for the Brusselator system and in the third section we will compare the approximate solutions obtained by using PLSM with the approximate solutions from [20]. The computations show that the approximations computed by using our method present an error smaller than the error of the corresponding solutions from [20]. 


\section{The Polynomial Least Squares Method}

For problem $((1),(2))$ we consider the remainder operators:

$$
\begin{aligned}
\mathfrak{D}_{1}(x(t), y(t))= & D_{t}^{\alpha_{1}} x(t) \\
& -\left[a-(\mu+1) \cdot x(t)+x(t)^{2} \cdot y(t)\right] \\
\mathfrak{D}_{2}(x(t), y(t))= & D_{t}^{\alpha_{2}} x(t)-\left[\mu \cdot x(t)-x(t)^{2} \cdot y(t)\right] .
\end{aligned}
$$

We will find approximate polynomial solutions $\widetilde{x}(t), \tilde{y}(t)$ of problem $((1),(2))$ on the $[0, b]$ interval, solutions which satisfy the following conditions:

$$
\begin{gathered}
\left|\mathfrak{D}_{j}(\tilde{x}(t), \tilde{y}(t))\right|<\epsilon, \quad j=1,2, \epsilon>0 \\
\tilde{x}(0)=c_{1}, \quad \tilde{y}(0)=c_{2} .
\end{gathered}
$$

Definition 1. One calls an $\epsilon$-approximate polynomial solution of problem ((1), (2)) an approximate polynomial solution $(\widetilde{x}(t), \tilde{y}(t))$ satisfying relations $((5),(6))$.

Definition 2. One calls a weak $\delta$-approximate polynomial solution of problem ((1), (2)) an approximate polynomial solution $(\tilde{x}(t), \tilde{y}(t))$ satisfying the relations:

$$
\int_{0}^{b} \mathfrak{D}_{j}^{2}(\tilde{x}(t), \tilde{y}(t)) d t \leq \delta, \quad j=1,2,
$$

together with initial conditions (6).

Definition 3. One considers the sequence of polynomials: $P_{m}^{j}(t)=a_{0}^{j}+a_{1}^{j} t+\cdots+a_{m}^{j} t^{m}, a_{i}^{j} \in \mathbb{R}, i=0,1, \ldots, m, j=1,2$, satisfying the conditions:

$$
P_{m}^{1}(0)=c_{1}, \quad P_{m}^{2}(0)=c_{2}, \quad m>1, \quad m \in \mathbb{N} .
$$

One calls the sequence of polynomials $P_{m}^{j}(t)$ convergent to the solution of problem $((1),(2))$ if $\lim _{m \rightarrow \infty} \mathfrak{D}_{j}\left(P_{m}^{1}(t)\right.$, $\left.P_{m}^{2}(t)\right)=0$.

We will find weak $\epsilon$-polynomial solutions of the type:

$$
\tilde{x}(t)=\sum_{k=0}^{m} d_{k}^{1} \cdot t^{k}, \quad \tilde{y}(t)=\sum_{k=0}^{m} d_{k}^{2} \cdot t^{k}, \quad m>1,
$$

where the constants $d_{0}^{j}, d_{1}^{j}, \ldots, d_{m}^{j}, j=1,2$ are calculated using the steps outlined as follows.

(i) We attach to problem ((1), (2)) the following real functional:

$$
J\left(d_{2}^{1}, \ldots, d_{m}^{1}, d_{2}^{2}, \ldots, d_{m}^{2}\right)=\sum_{j=1}^{2} \int_{0}^{b} \mathfrak{D}_{j}^{2}(\tilde{x}(t), \tilde{y}(t)) d t
$$

where $d_{0}^{1}, d_{0}^{2}$ are computed as functions of $d_{1}^{1}, d_{2}^{1}, \ldots$, $d_{m}^{1}, d_{1}^{2}, d_{2}^{2}, \ldots, d_{m}^{2}$ by using initial conditions (6). (ii) We computes the values of $\bar{d}_{1}^{1}, \bar{d}_{2}^{1}, \ldots, \bar{d}_{m}^{1}, \bar{d}_{1}^{2}, \bar{d}_{2}^{2}, \ldots$, $\bar{d}_{m}^{2}$ as the values which give the minimum of functional (10) and the values of $\bar{d}_{0}^{1}, \bar{d}_{0}^{2}$ again as functions of $\bar{d}_{1}^{1}, \bar{d}_{2}^{1}, \ldots, \bar{d}_{m}^{1}, \bar{d}_{1}^{2}, \bar{d}_{2}^{2}, \ldots, \bar{d}_{m}^{2}$ by using the initial conditions.

(iii) Using the constants $\bar{d}_{0}^{1}, \bar{d}_{1}^{1}, \ldots, \bar{d}_{m}^{1}, \bar{d}_{0}^{2}, \bar{d}_{1}^{2}, \ldots, \bar{d}_{m}^{2}$ thus determined, we consider the polynomials:

$$
T_{m}^{1}(t)=\sum_{k=0}^{m} \bar{d}_{k}^{1} \cdot t^{k}, \quad T_{m}^{2}(t)=\sum_{k=0}^{m} \bar{d}_{k}^{2} \cdot t^{k}, \quad m>1 .
$$

The following convergence theorem holds.

Theorem 4. The necessary condition for problem ((1), (2)) to admit sequences of polynomials $P_{m}^{j}(t)$ convergent to the solution of this problem is

$$
\lim _{m \rightarrow \infty} \int_{0}^{b} \mathfrak{D}_{j}^{2}\left(T_{m}^{1}(t), T_{m}^{2}(t)\right) d t=0 .
$$

Moreover, $\forall \epsilon>0, \exists m_{0} \in \mathbb{N}$, such that, $\forall m \in \mathbb{N}, m>$ $m_{0}$, it follows that $T_{m}^{j}(t), j=1,2$, are weak $\epsilon$-approximate polynomial solutions of problem ((1), (2)).

Proof. Based on the way the coefficients of the polynomials $T_{m}^{j}(t)$ are computed and taking into account relations ((9)(11)), the following inequality holds:

$$
\begin{aligned}
0 & \leq \int_{0}^{b} \mathfrak{D}_{j}^{2}\left(T_{m}^{1}(t), T_{m}^{2}(t)\right) d t \\
& \leq \int_{0}^{b} \sum_{j=1}^{n} \mathfrak{D}_{j}^{2}\left(P_{m}^{1}(t), P_{m}^{2}(t)\right) d t, \quad \forall m \in \mathbb{N} .
\end{aligned}
$$

It follows that

$$
\begin{aligned}
0 & \leq \lim _{m \rightarrow \infty} \int_{0}^{b} \mathfrak{D}_{j}^{2}\left(T_{m}^{1}(t), T_{m}^{2}(t)\right) d t \\
& \leq \lim _{m \rightarrow \infty} \int_{0}^{b} \sum_{j=1}^{n} \mathfrak{D}_{j}^{2}\left(P_{m}^{1}(t), P_{m}^{2}(t)\right) d t=0, \quad \forall m \in \mathbb{N} .
\end{aligned}
$$

We obtain

$$
\lim _{m \rightarrow \infty} \int_{0}^{b} \mathfrak{D}_{j}^{2}\left(T_{m}^{1}(t), T_{m}^{2}(t)\right) d t=0
$$

From this limit we obtain that, $\forall \epsilon>0, \exists m_{0} \in \mathbb{N}$ such that, $\forall m \in \mathbb{N}, m>m_{0}$, it follows that $T_{m}^{j}(t)$ are weak $\epsilon$ approximate polynomial solutions of problem ((1), (2)), $j=$ 1,2 .

Remark 5. Any $\epsilon$-approximate polynomial solutions of problem $((1),(2))$ are also weak $\epsilon^{2} \cdot b$-approximate polynomial solutions, but the opposite is not always true. It follows that the set of weak approximate solutions of problem $((1),(2))$ also contains the approximate solutions of the system. 


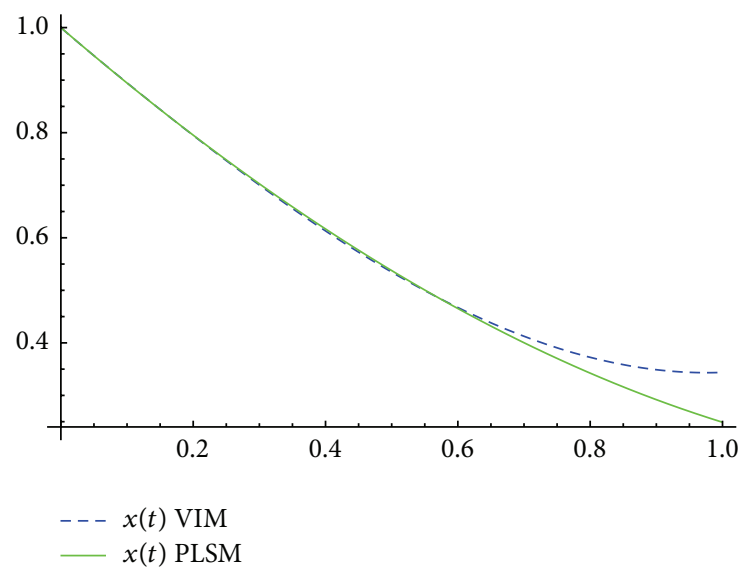

Figure 1: Approximations for $x(t)$ in the case $\alpha_{1}=\alpha_{2}=0.98$.

Taking into account the above remark, in order to find $\epsilon$-approximate polynomial solutions of problem ((1), (2)) by PLSM we will first determine weak approximate polynomial solutions, $\widetilde{x}(t), \tilde{y}(t)$. If $\left|\mathfrak{D}_{j}(\widetilde{x}(t), \tilde{y}(t))\right|<\epsilon, j=1,2$, then $\widetilde{x}(t), \tilde{y}(t)$ are also $\epsilon$-approximate polynomial solutions of the system.

\section{Application: the Fractional-Order Brusselator System}

We consider the following fractional-order Brusselator system [20]:

$$
\begin{gathered}
D_{t}^{\alpha_{1}} x(t)=-2 \cdot x(t)+x(t)^{2} \cdot y(t), \\
D_{t}^{\alpha_{2}} y(t)=x(t)-x(t)^{2} \cdot y(t),
\end{gathered}
$$

together with the initial conditions:

$$
x(0)=1, \quad y(0)=1 .
$$

In [20] approximate solutions of (17) are computed using the Variational Iteration Method (VIM) for the case $\alpha_{1}=$ $\alpha_{2}=0.98$. Also, a comparison with numerical solutions is presented for the particular case $\alpha_{1}=\alpha_{2}=1$, illustrating the applicability of the method.

3.1. The Case $\alpha_{1}=\alpha_{2}=0.98$. For the case $\alpha_{1}=\alpha_{2}=0.98$, using PLSM with $m=3$, we obtain the following approximate polynomial solutions:

$$
\begin{aligned}
& x_{\mathrm{PLSM}}(t)=0.0243682 \cdot t^{3}+0.311138 \cdot t^{2}-1.08655 \cdot t+1 \\
& y_{\mathrm{PLSM}}(t)=-0.184414 \cdot t^{3}+0.333424 \cdot t^{2} \\
&+0.0349127 \cdot t+1 .
\end{aligned}
$$

In Figures 1 and 2 we compare these approximations with the corresponding approximations of the same order computed by VIM (relations (15) in [20]), obtaining a good

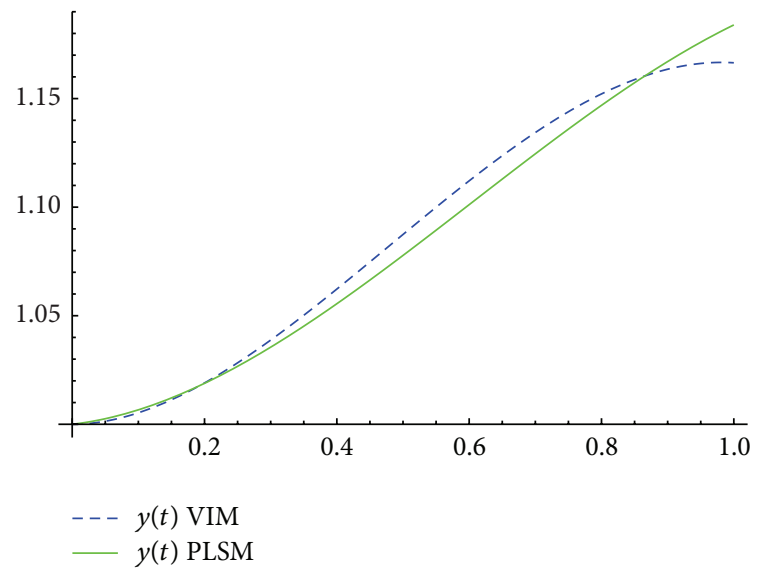

Figure 2: Approximations for $y(t)$ in the case $\alpha_{1}=\alpha_{2}=0.98$.

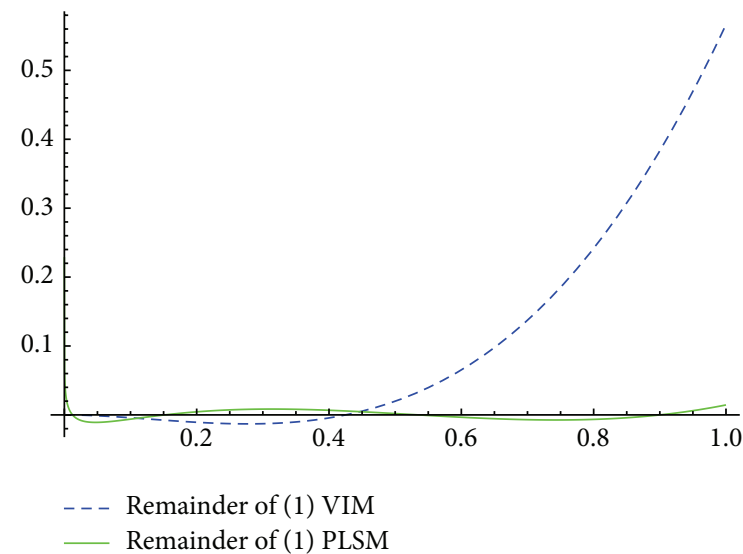

FIGURE 3: The remainders corresponding to the first equation for the case $\alpha_{1}=\alpha_{2}=0.98$.

agreement. In Figures 3 and 4 we compare the expressions of remainders (4) obtained by replacing the approximate solutions back in the equations. It is easy to observe that the errors obtained by using PLSM are smaller than the ones obtained by using VIM.

3.2. The Case $\alpha_{1}=\alpha_{2}=1$. For the case $\alpha_{1}=\alpha_{2}=1$, using PLSM with $m=3$ we obtain the following approximate polynomial solutions:

$$
\begin{gathered}
x_{\text {PLSM }}(t)=0.0750974 \cdot t^{3}+0.201028 \cdot t^{2}-1.02827 \cdot t+1 \\
y_{\text {PLSM }}(t)=-0.180088 \cdot t^{3}+0.334087 \cdot t^{2} \\
+0.0271107 \cdot t+1 .
\end{gathered}
$$

In this case both approximations (VIM and PLSM) consist of third-order polynomials.

We omitted the figures which compare our approximations with the ones given by VIM since they look almost the same as the corresponding ones from the case $\alpha_{1}=\alpha_{2}=0.98$.

However, in the case $\alpha_{1}=\alpha_{2}=1$ it is possible to compute the absolute error corresponding to an approximate solution 


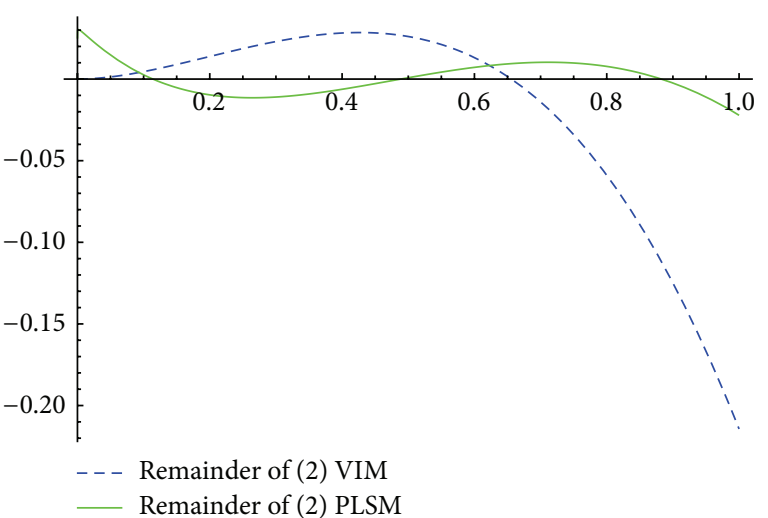

FIGURE 4: The remainders corresponding to the second equation for the case $\alpha_{1}=\alpha_{2}=0.98$.

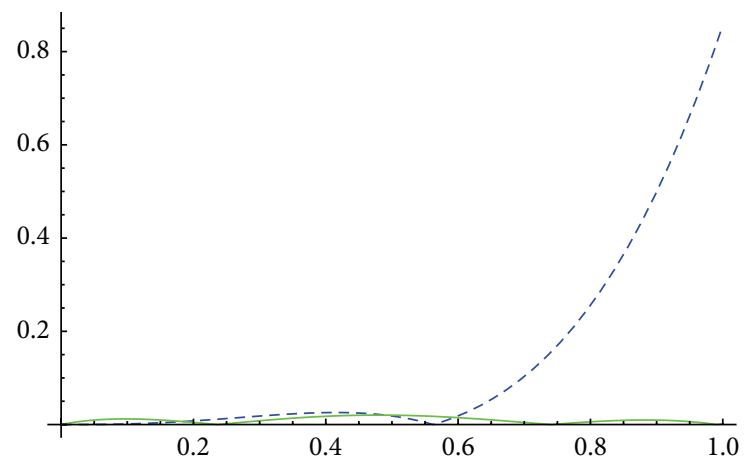

- - - Absolute error of $x(t)$ VIM

_ Absolute error of $x(t)$ PLSM

FigURE 5: The absolute errors corresponding to $x(t)$ for the case $\alpha_{1}=$ $\alpha_{2}=1$.

as the difference in absolute value between the approximate solution and the numerical solution (in this case computed by using the Wolfram Mathematica software).

Figures 5 and 6 present the comparison between the absolute errors corresponding to the approximate solutions from [20] obtained by VIM and the absolute errors corresponding to our approximate solutions.

Again, it is easy to observe that the errors obtained by using PLSM are smaller than the ones obtained by using VIM.

\section{Conclusions}

In this paper we present the Polynomial Least Squares Method, which is a relatively straightforward and efficient method to compute approximate solutions for the fractionalorder Brusselator system.

The comparison with previous results illustrates the accuracy of the method, since we were able to compute more precise approximations than the previously computed ones.

In closing we mention the fact that, due to the nature of the method, it is relatively easy to extend PLSM for the general

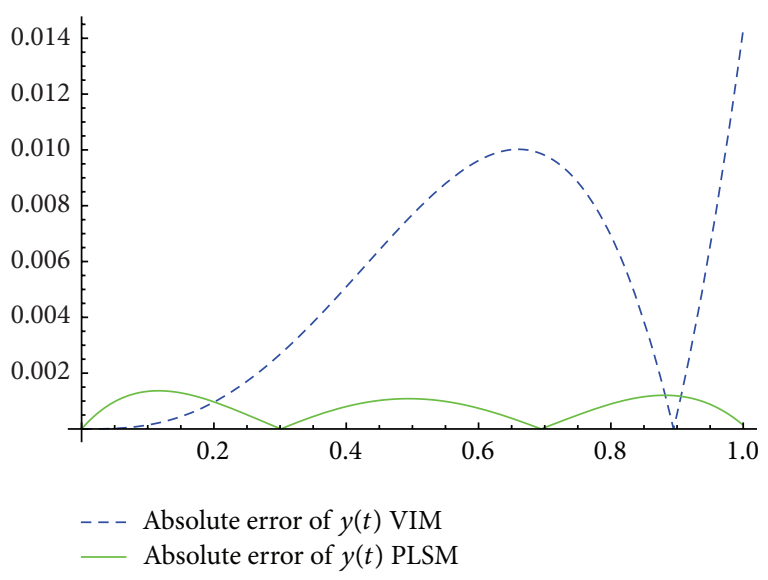

FIGURE 6: The absolute errors corresponding to $y(t)$ for the case $\alpha_{1}=$ $\alpha_{2}=1$.

case of fractional systems of $n \geq 3$ nonlinear differential equations.

\section{Conflict of Interests}

The authors declare that there is no conflict of interests regarding the publication of this paper.

\section{References}

[1] R. Hilfer, Applications of Fractional Calculus in Physics, Academic Press, Orlando, Fla, USA, 1999.

[2] A. M. Spasic and M. P. Lazarevic, "Electroviscoelasticity of liquid/liquid interfaces: fractional-order model," Journal of Colloid and Interface Science, vol. 282, no. 1, pp. 223-230, 2005.

[3] F. Mainardi, "Fractional calculus: some basic problems in continuum and statistical mechanics," in Fractals and Fractional Calculus in Continuum Mechanics, A. Carpinteri and F. Mainardi, Eds., pp. 291-348, Springer, New York, NY, USA, 1997.

[4] A. Carpinteri and F. Mainardi, Fractals and Fractional Calculus in Continuum Mechanics, Springer, New York, NY, USA, 1997.

[5] R. Goreno and F. Mainardi, "Fractional calculus: integral and differential equations of fractional order," in Fractals and Fractional Calculus in Continuum Mechanics, A. Carpinteri and F. Mainardi, Eds., pp. 223-276, Springer, New York, NY, USA, 1997.

[6] A.M. Nagy and N.H. Sweilam, "An efficient method for solving fractional Hodgkin-Huxley model," Physics Letters A, vol. 378, no. 30-31, pp. 1980-1984, 2014.

[7] V. Daftardar-Gejji and H. Jafari, "Adomian decomposition: a tool for solving a system of fractional differential equations," Journal of Mathematical Analysis and Applications, vol. 301, no. 2, pp. 508-518, 2005.

[8] H. Jafari and V. Daftardar-Gejji, "Solving a system of nonlinear fractional differential equations using Adomian decomposition," Journal of Computational and Applied Mathematics, vol. 196, no. 2, pp. 644-651, 2006.

[9] M. Zurigat, "Solving nonlinear fractional differential equation using a multi-step Laplace adomian decomposition method," Annals of the University of Craiova, Mathematics and Computer Science Series, vol. 39, no. 2, pp. 200-210, 2012. 
[10] H. Jafari and S. Seifi, "Homotopy analysis method for solving linear and nonlinear fractional diffusion-wave equation," Communications in Nonlinear Science and Numerical Simulation, vol. 14, no. 5, pp. 2006-2012, 2009.

[11] L. Song and H. Zhang, "Application of homotopy analysis method to fractional KdV-Burgers-Kuramoto equation," Physics Letters A, vol. 367, no. 1-2, pp. 88-94, 2007.

[12] M. Zurigat, S. Momani, Z. Odibat, and A. Alawneh, "The homotopy analysis method for handling systems of fractional differential equations," Applied Mathematical Modelling, vol. 34, no. 1, pp. 24-35, 2010.

[13] Z. Odibat and S. Momani, "Modified homotopy perturbation method: application to quadratic Riccati differential equation of fractional order," Chaos, Solitons and Fractals, vol. 36, no. 1, pp. 167-174, 2008.

[14] S. Momani and Z. Odibat, "Numerical approach to differential equations of fractional order," Journal of Computational and Applied Mathematics, vol. 207, no. 1, pp. 96-110, 2007.

[15] I. Podlubny, Fractional Differential Equations, Academic Press, San Diego, Calif, USA, 1999.

[16] K. S. Miller and B. Ross, An Introduction to the Fractional Calculus and Fractional Differential Equations, Wiley, New York, NY, USA, 1993.

[17] S. Kemle and H. Beyer, "Global and causal solutions of fractional differential equations," in Proceedings of the 2nd International Workshop on Transform Methods and Special Functions (SCTP '97), pp. 210-216, Singapore, 1997.

[18] S. Dixit, O. P. Singh, and S. Kumar, "An analytic algorithm for solving system of fractional differential equations," Journal of Modern Methods in Numerical Mathematics, vol. 1, no. 1, pp. 1226, 2010.

[19] X.-J. Yang and D. Baleanu, "Fractal heat conduction problem solved by local fractional variation iteration method," Thermal Science, vol. 17, no. 2, pp. 625-628, 2013.

[20] H. Jafari, A. Kadem, and D. Baleanu, "Variational iteration method for a fractional-order Brusselator system," Abstract and Applied Analysis, vol. 2014, Article ID 496323, 6 pages, 2014.

[21] Y. Wang and C. Li, "Does the fractional Brusselator with efficient dimension less than 1 have a limit cycle?" Physics Letters A, vol. 363, no. 5-6, pp. 414-419, 2007.

[22] V. Gafiychuk and B. Datsko, "Stability analysis and limit cycle in fractional system with Brusselator nonlinearities," Physics Letters, Section A: General, Atomic and Solid State Physics, vol. 372, no. 29, pp. 4902-4904, 2008.

[23] S. Kumar, Y. Khan, and A. Yildirim, "A mathematical modeling arising in the chemical systems and its approximate numerical solution," Asia-Pacific Journal of Chemical Engineering, vol. 7, no. 6, pp. 835-840, 2012. 


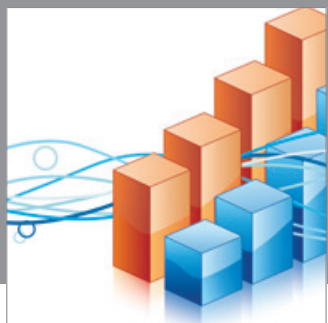

Advances in

Operations Research

mansans

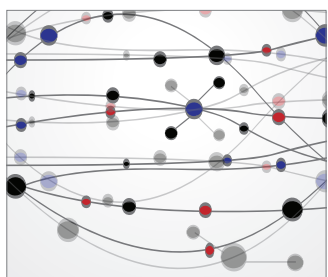

The Scientific World Journal
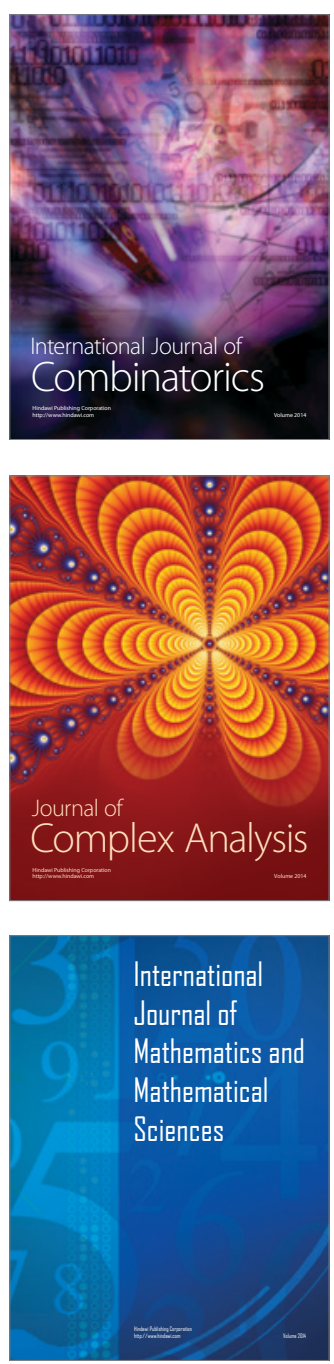
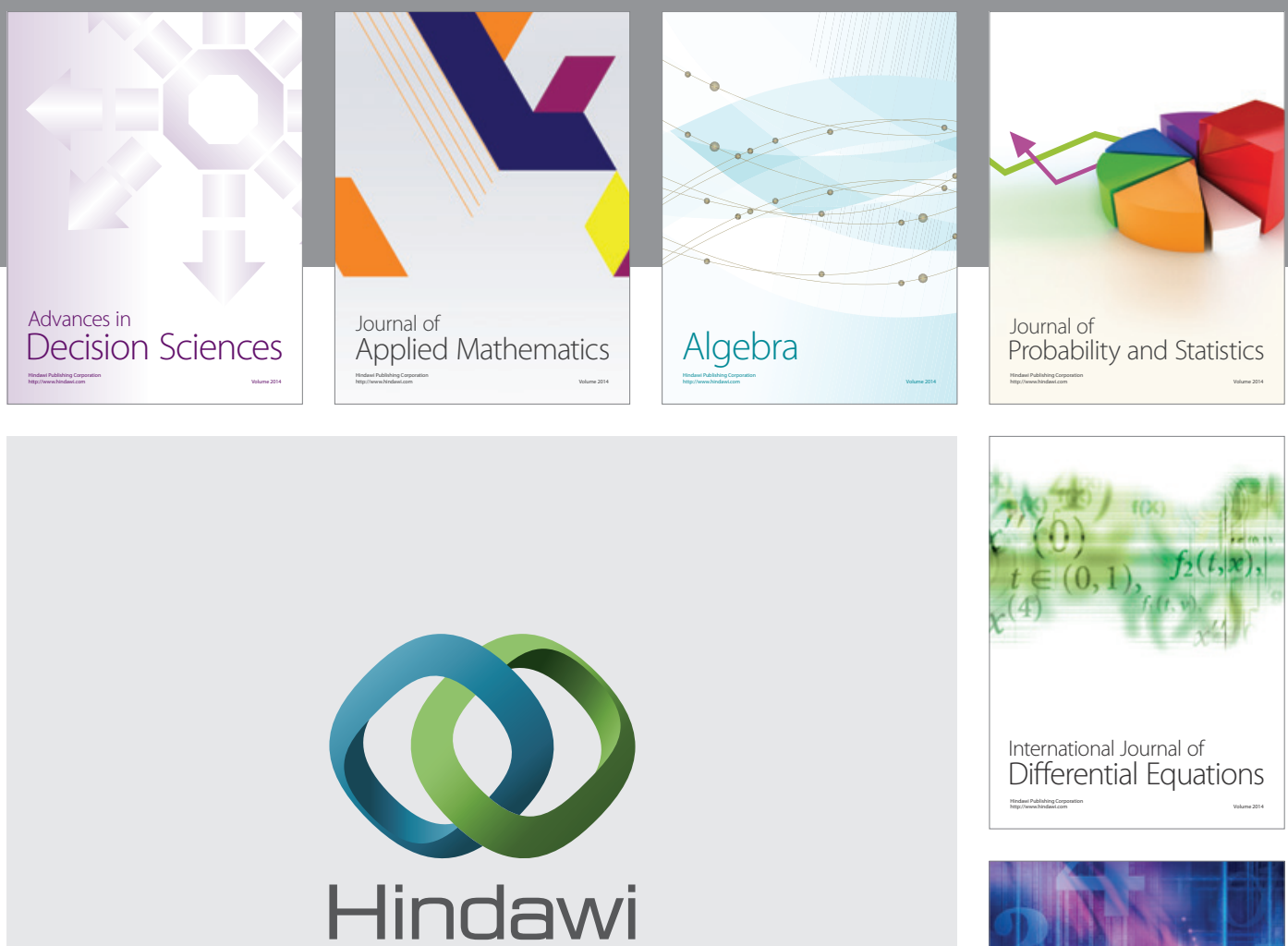

Submit your manuscripts at http://www.hindawi.com
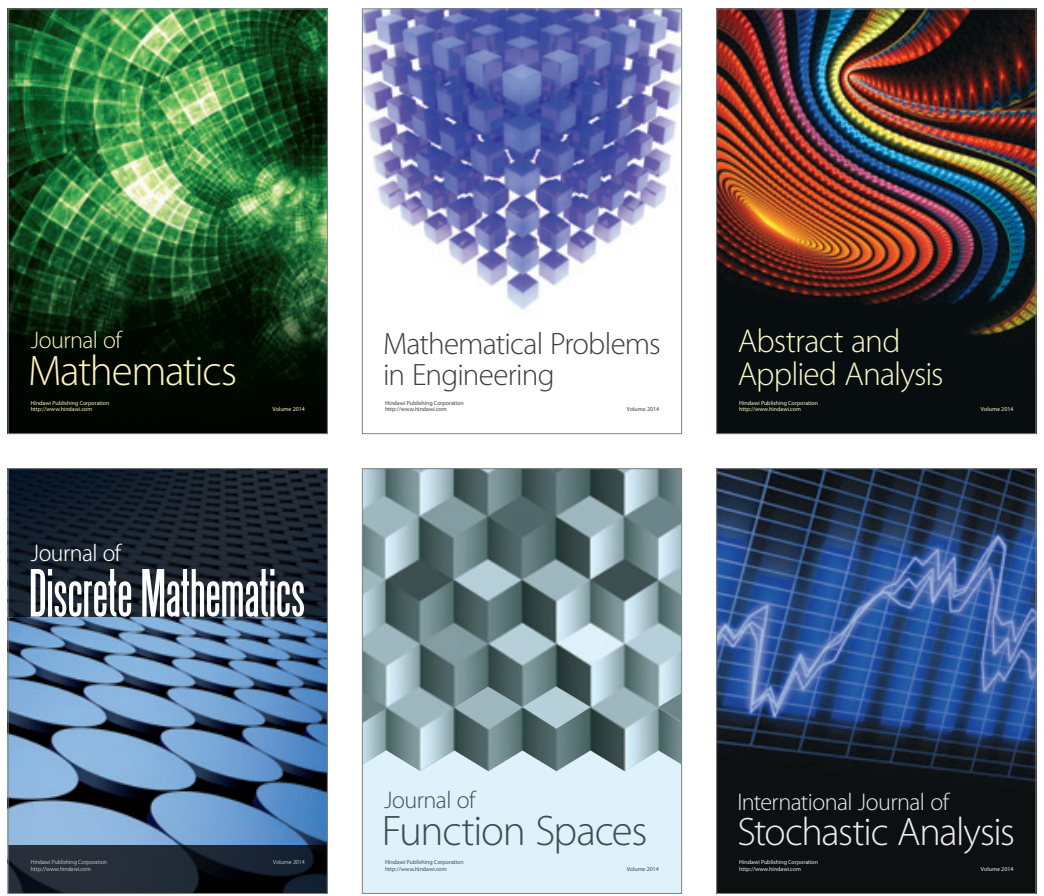

Journal of

Function Spaces

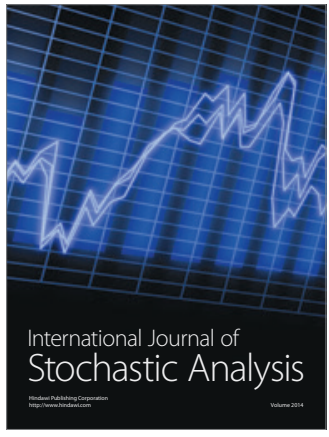

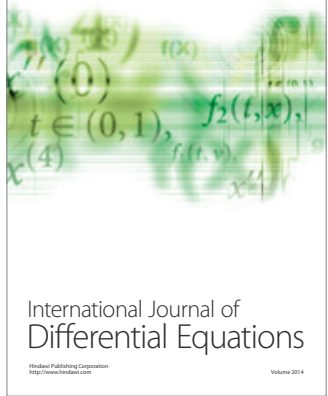
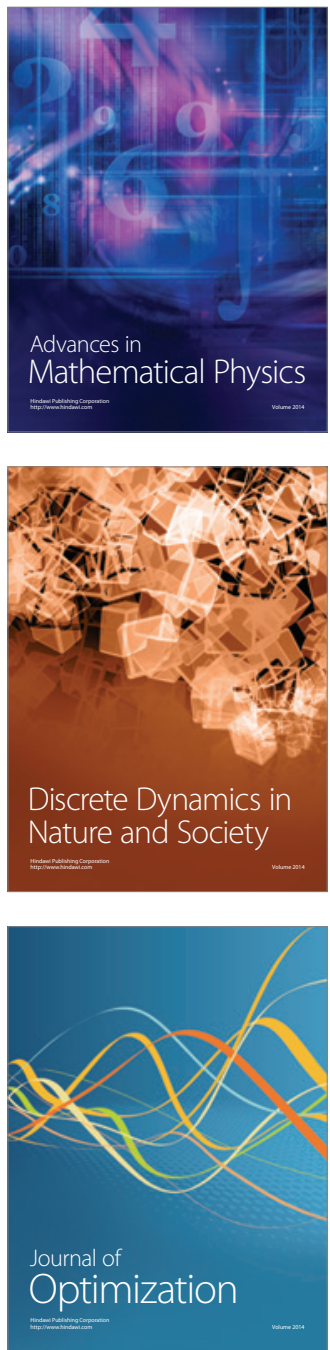